\title{
Treatment Strategies for Cannabis Use in Schizophrenia
}

Brian R. Schultz, M.D., Ph. $D^{1}$

Lisette Rodriguez-Cabezas, M.D $D^{1}$

Dan Angres, M. $D^{1,2}$

Matthew J. Smith, Ph. $D^{1,3, *}$

\author{
Address \\ ${ }^{*}, 1$ Department of Psychiatry and Behavioral Sciences, Northwestern University \\ Feinberg School of Medicine, 446 East Ontario Street, Suite 7-100, Chicago, IL \\ 60611, USA \\ Email: matthewsmith@northwestern.edu \\ ${ }^{2}$ Positive Sobriety Institute, 680 N. Lake Shore Drive, Suite 800 , Chicago, IL \\ 60611, USA \\ ${ }^{3}$ Warren Wright Adolescent Center, Northwestern University Feinberg School of \\ Medicine, 710 N. Lake Shore Drive, 13th Floor, Chicago, IL 60611, USA
}

Published online: 8 April 2015

(C) Springer International Publishing AG 2015

This article is part of the Topical Collection on Schizophrenia and Other Psychotic Disorders

Keywords Schizophrenia - Psychosis - Cannabis use disorder - Psychosocial treatment - Pharmacological treatment

\section{Opinion statement}

Cannabis is used by more than $25 \%$ of schizophrenia patients and is associated with symptom exacerbation and poorer clinical outcome. To date, evidence is scarce for treating cannabis use disorders among schizophrenia patients. Psychosocial interventions such as cognitive behavioral therapy, motivational interviewing, or contingency management have been evaluated as potential treatments for cannabis use disorders (CUD) among schizophrenia patients and in the general population. However, results are somewhat inconsistent, and efficacy appears to wane after treatment concludes. Pharmacotherapy has also been examined as a potential treatment for CUD but has not yet demonstrated consistent efficacy in the general population. The administration of second-generation antipsychotic medications is the pharmacological standard of care for schizophrenia as well as the treatment of choice for comorbid cannabis use among schizophrenia patients. Recent preliminary trials of pharmacotherapy agents in the general population must be further tested to confirm their potential efficacy at treating CUD. Based on the current evidence, we recommend a multi-faceted approach to treatment for CUD in the schizophrenia population, employing pharmacological and psychosocial modalities. We recommend using a second-generation antipsychotic medication; to date risperidone, 
olanzapine, and clozapine show the most promise for this population. We also recommend engaging patients in a combination of motivation-based treatment (motivational interviewing or motivation enhancement therapy) and cognitive behavioral treatment directed at cannabis use. When family systems allow, multidimensional family therapy should also be considered, particularly for adolescent patients. The available evidence suggests that CUDs will require ongoing treatment in order to maintain long-term efficacy.

\section{Introduction}

Up to $50 \%$ of schizophrenia patients meet criteria for a comorbid substance use disorder at some time during their lives [1]. These individuals tend to be male [2], younger at symptom onset [3], experience greater extrapyramidal and depressive symptoms [4,5], have less severe negative symptoms [6], and more severe positive symptoms [2]. Overall, they have poor treatment adherence [7], a lower quality of life [8], exhibit more violent behavior [9], and are more likely to be homeless [10] and unemployed [5]. Despite these poorer outcomes, clinical trials to assess treatments for schizophrenia often exclude patients with substance use disorders and trials to treat substance use disorders often exclude schizophrenia patients [11].

This scientific approach leaves a large gap in our knowledge of how to best treat this patient population [11]. Hence, there is a specific need to develop treatments for schizophrenia patients with comorbid substance use disorders. Cannabis is the most commonly used illicit substance by schizophrenia patients as prevalence rates range from 27 to $42 \%[12,13]$. To date, studies suggest that cannabis use leads to reduced treatment compliance [14-18], increased re-hospitalizations and relapses [19-21], and greater severity in psychotic symptoms $[12,22]$. Of particular significance is the damaging effect that cannabis use disorders may have on the brain, based on evidence that cannabis use contributed to greater progressive cortical thinning over 5 years among a comorbid cannabis-using schizophrenia group compared to non-using schizophrenia patients and controls [23], and that abusing during adolescence is associated with persistent differences in subcortical morphology among schizophrenia patients after extended abstinence [24].

Although there is a large literature suggesting that cannabis use is detrimental to the clinical course of schizophrenia [25], there are few evidence-based treatments available that demonstrate efficacy or effectiveness at treating comorbid cannabis use disorders among schizophrenia patients. Hence, our objective is to review the most recent evidence available for treating cannabis use disorders among schizophrenia patients. Of note, the majority of the reviewed studies used diagnostic criteria consistent with cannabis abuse or dependence defined by DSM-IV [26]. We will use the DSM-V term "cannabis use disorder" (CUD) as a term to encompass both abuse and dependence in this review [27]. We searched PubMed, PsychInfo, Social Sciences Abstracts, and the Cochrane Database of Systematic Reviews to find peerreviewed publications reporting on the specific treatment of CUDs from 1994 to 2014.

\section{Treating cannabis use disorders among schizophrenia patients}

Few studies have specifically investigated the treatment of CUDs among schizophrenia patients. A systematic review of this topic only found 11 studies of cannabis use treatment as a separate outcome distinct from other substance use disorders [28]. The authors concluded that there was insufficient evidence to support the efficacy or effectiveness of treatments for CUD among schizophrenia patients. However, in studies with grouped substance use, there were positive outcomes that psychosocial interventions and pharmacological treatment may confer some benefit at reducing cannabis use [28]. 
The most commonly studied psychosocial interventions discussed in this review are motivational interviewing (MI), cognitive behavioral therapy (CBT), and contingency management (CM). MI is a counseling style that aims to resolve ambivalence and allows patients to change through a selfactualization process [29]. CBT, originally developed to treat depression [30], has been adapted into many forms to treat a variety of disorders and is based on developing cognitive change in a patient that leads to changes in the patient's thought process and belief system, subsequently leading to emotional and behavioral changes [31]. $\mathrm{CM}$ is based on providing rewards to patients to reinforce positive behaviors, such as abstinence, attendance, or completing homework [32].

A systematic review of 32 randomized control trials (RCTs) of psychosocial interventions for patients with severe mental illness and comorbid substance abuse did not find compelling evidence to support any one psychosocial treatment modality over another regarding treatment retention, substance use reduction, or symptom improvement [33•]. Furthermore, the review did not find evidence that active treatment groups fared better than treatment-as-usual (TAU) control groups in studies investigating long-term integrated care; nonintegrated intensive case management; MI, CBT, MI and CBT together; skills training; or CM. The observed lack of group differences were explained by limitations in study design, outcome measures, treatment fidelity, and high attrition rates [33•].

Although most studies in this systematic review targeted the treatment of alcohol or illicit substances in general, a few studies focused on treating cannabis use among patients with a schizophrenia spectrum disorder (e.g., schizophrenia, schizoaffective disorder, schizophreniform, psychosis not otherwise specified) [33•]. Specifically, an RCT evaluating 62 patients with a schizophrenia spectrum disorder ( $<35$ years old) found that 6 months of treatment with MI was associated with a reduction in the number of joints smoked per week at 3 and 6 months while receiving treatment, but the effects were not sustained at a 12-month post-treatment follow-up [34].

A multi-center RCT of 88 patients with a schizophrenia spectrum disorder performed a group-based psychological intervention based on CBT and MI weekly for 12 weeks, plus a "booster" session 6 weeks later and did not find differences in cannabis use compared to TAU at 3 and 12 months posttreatment follow-up [35]. A recent RCT of 103 schizophrenia spectrum disorder patients with CUDs evaluated the efficacy of MI+CBT compared to TAU and reported that MI+CBT participants self-reported smoking fewer joints per day than TAU participants, but the number of days they smoked was not reduced after 6 months of treatment [36]. A secondary analysis of this study found that, over a 3-year period, MI+CBT participants had fewer days of hospitalization but had greater risk of psychiatric emergency department contact and more admissions to psychiatric hospitals compared to TAU participants [37]. These findings suggest that MI+CBT participants may be more likely to seek treatment, which could explain more contact with treatment providers and fewer days in observed care [37]. Another RCT examined using family-based MI (FMI) and interaction skills training among the parents of patients $(n=75)$ with recentonset schizophrenia and co-occurring cannabis use. Parents $(n=97)$ were 
randomly allocated to the FMI group or to a routine family support (RFS) group; each group met every other week for 12 weeks. There was a significant decrease in self-reported number of days using cannabis, total grams used, and craving of cannabis observed between assessments at 3 months pre-treatment and 3 months post-treatment. However, there was no significant between-group difference in the proportion of negative urine tests at follow-up [38].

Lastly, a recent study queried 120 schizophrenia patients about their strategies for quitting cannabis use and for relieving cannabis withdrawal symptoms [39]. Nearly $96 \%$ of patients used at least one strategy to maintain abstinence during their attempt to quit using cannabis. The most common strategies were (1) getting rid of cannabis or paraphernalia, (2) ending their association with others who smoke cannabis, and (3) avoiding places where cannabis is used. Moreover, the strategy rated as "most helpful" was receiving support from religious services or through prayer [39]. Overall, there appears to be some benefit conferred by psychosocial treatments for cannabis use among schizophrenia patients. However, the evidence concerning long-term efficacy remains sparse and inconsistent and, as such, requires additional research to clarify the direction of these findings.

Recent pharmacological studies examining treatment for CUDs in schizophrenia patients focused on the effects of second-generation antipsychotic medications, which are also the first-line choice for treating psychosis [40, 41]. For example, two studies found decreased substance use among schizophrenia spectrum patients after treatment with quetiapine or clozapine [42, 43]. In regard to specifically treating CUDs, a 6-week double-blinded RCT in 128 patients with schizophrenia spectrum disorders compared treatment with olanzapine vs. risperidone and found a similar decrease in cannabis craving between the two groups [44]. More recently, a randomized open-label prospective study of first-episode patients diagnosed with schizophrenia spectrum disorders $(n=49)$ compared the effects of treatment with olanzapine vs. risperidone on cannabis use over 16 weeks but yielded no between-group differences regarding rates of cannabis use [45].

A randomized trial compared the effects of risperidone and clozapine on cannabis craving and associated regional brain activity in 28 schizophrenia patients with comorbid CUDs [46•]. After 4 weeks of treatment, the clozapine group showed larger reductions in cannabis craving and activation in the insula during a cannabis word Stroop test when compared to the group treated with risperidone [46•]. Another study evaluated cannabis craving among 503 patients with schizophrenia spectrum disorders and comorbid CUDs treated with risperidone, olanzapine, or clozapine [47]. The results suggested that patients using risperidone had significantly more cannabis craving than those treated with olanzapine or clozapine. There was no significant difference in craving between the olanzapine and clozapine groups [47].

In summary, there is minimal evidence supporting the use of antipsychotic medications to treat CUDs among schizophrenia patients as recent uncontrolled trials produced inconsistent results regarding reductions in craving and cannabis use. Notable trends indicated that olanzapine and clozapine might be 
more efficacious for treatment of cannabis cravings than risperidone, consistent with the drugs' comparative efficacy of treating schizophrenia alone $[48,49]$.

\section{Treatment in the general population}

Most studies of CUD treatment exclude patients with a co-existing mental illness such as schizophrenia. Thus, we discuss the findings from treatment studies that focused on CUDs among individuals who are otherwise psychiatrically healthy. We expect this review will identify interventions that can be evaluated among schizophrenia patients.

Interventions that are similar to those tested among schizophrenia patients have been investigated more frequently as treatments for CUD in the general population [50-58]. These included brief or extended CBT, motivational enhancement therapy (MET) [32], and CM or a combination thereof. Most studies combined treatment modalities in an effort to reduce cannabis use. For example, a recent trial randomized 215 patients diagnosed with CUD to 9 weeks treatments of (1) MET+CBT+CM-for-completing-homework, (2) $\mathrm{MET}+\mathrm{CBT}+\mathrm{CM}$-for-abstinence, or (3) case management. For the primary outcome measures of continued abstinence and proportion of days abstinent, no significant differences were found when comparing the case management group to the other two conditions (although a modest decrease in cannabis use was observed across all groups at 14 months follow-up) [59]. This finding suggests that professional contact alone is associated with decreased cannabis use. Also, a subsample of patients treated with $\mathrm{MET}+\mathrm{CBT}+\mathrm{CM}$-for-abstinence became long-term abstainers [59].

A 12-week study of 127 treatment-seeking youths from the criminal justice system evaluated the efficacy of combining CBT with CM (CBT+CM) to improve abstinence [60]. Participants were randomized to four groups: (1) CBT alone, (2) CBT+CM reinforcing homework and attendance, (3) CM for abstinence, or (4) $\mathrm{CBT}+\mathrm{CM}$ for abstinence. The results demonstrated that the $\mathrm{CBT}+\mathrm{CM}$ reinforcing homework and attendance treatment did not significantly improve abstinence over CBT alone after 12 weeks sessions, while the CBT+CM for abstinence group produced a higher proportion of cannabis-positive urine specimens compared to CM alone. At 12 months follow-up, CBT alone and CM alone had a similar frequency and also the lowest frequency of cannabis use among the four treatment groups [60]. This finding was unexpected, considering previous trials suggested that adding CM to MET or CBT increased treatment adherence or reduced cannabis use [50-52]. The negative findings could be explained by the provision of rewards through CM being met with reactance by youth from the juvenile justice system [60].

The Cannabis Disorders (CANDIS) trial evaluated the efficacy of a combination of MET, CBT, and psychosocial problem-solving training at treating CUDs [61]. CANDIS randomized 122 patients with CUD to treatment with ten 90 min twice-weekly sessions (over 5-8 weeks) or a delayed treatment group who were instructed to reduce or discontinue cannabis use. The treatment group abstinence rate was $49 \%$ at the end of treatment and $41.1 \%$ at 
6 months follow-up. In contrast, delayed treatment group abstinence rate was $12.5 \%$ after the 8 weeks delay prior to treatment [61]. A follow-up RCT using the CANDIS protocol randomized 279 patients to active treatment or delayed treatment $[62 \bullet \bullet]$. The rate of negative drug screenings at the end of 10 sessions over 8-12 weeks, increased from 11.7 to $46.3 \%$ in the active treatment arm, compared to an increase from 9.3 to $17.7 \%$ in the delayed treatment group after 8 weeks (prior to beginning treatment). At 6 months follow-up, the treatment group's negative drug screen rate decreased from 46.3 to $35.7 \%$ $[62 \bullet \bullet]$.

Another RCT of cannabis-using high school youths $(n=310)$ compared the treatment effects of MET to an education feedback control (EFC) group and a delayed feedback control (DFC) group [63]. The MET group received two 4550-min sessions, the EFC group was shown a presentation on current research and facts about cannabis, and the DFC group received no treatment for 3 months. The MET and EFC groups received up to four CBT sessions after the initial treatments. At 3 months follow-up, both the MET and EFC group reported fewer days of cannabis use since completing treatment compared to the DFC group over the same period of time. The effect between MET and EFC was maintained at 12 months follow-up; however, the magnitude of change was modest-when asked the number of days used over the prior 60 days, the MET group had decreased from 40.23 days at baseline to 33.71 days at 12 months and the EFC group had decreased from 37.69 to 34.24 days.

Multidimensional family therapy (MDFT) is a family-based therapy designed to treat adolescent substance use within the framework that adolescent drug abuse is related to many life domains (e.g., biological, social, cognitive, interpersonal, familial, developmental, etc.) and that family functioning is important to foster healthier alternatives [64]. The efficacy of MDFT compared to CBT at reducing cannabis use was studied in a 26 week RCT consisting of 109 adolescents with CUD living in the Netherlands [65]. MDFT and CBT demonstrated similar magnitudes in post-treatment changes such as fewer days using cannabis, reduced frequency of using cannabis, and reduced delinquent behavior. Additionally, the authors found that 17-18 year-old participants benefitted more from CBT than from MDFT, while 13-16 year olds benefitted more from MDFT than CBT [66]. A 12 month RCT compared MDFT (two sessions per week) to individual psychotherapy (not standardized across sites) for treating CUDs in 13-18 year olds [67•]. They observed that the MDFT groups had a greater rate of decline in cannabis dependence from 82 to $38 \%$ compared to 82 to $52 \%$ in the individual psychotherapy group [67•].

There have also been a series of studies evaluating a brief intervention approach to treat CUDs. One RCT comparing one CBT session, six CBT sessions, and a delayed treatment control (DTC) group showed significant decrease in daily cannabis use in the six-session group compared to the DTC after approximately 8 months of follow-up, but no difference between the one-session group and DTC [56]. Another RCT compared two MET sessions, nine multicomponent therapy sessions (MET + CBT + case management), and a DTC group, finding a significant decrease at 4 months in the percentage of days of cannabis use between the two-session group and the DTC and between the nine-session group and the DTC. They also found a significantly higher rate of abstinence in the nine-session group compared to the other two groups [53]. A third RCT compared the efficacy of a 2-session individualized MI treatment, a 
14-session cognitive behavioral group treatment, and a DTC group at treating cannabis use. They observed a significant decrease in the number of days using cannabis per month at 4 months follow-up between either treatment group and the DTC group and a significant decrease in cannabis use by 16 months followup compared to baseline [58].

A more recent RCT evaluated the efficacy of using either written or oral psychoeducation to reduce cannabis use. Participants ( $n=134$ undergraduates) were randomized to one of four groups. Two treatment groups were provided cannabis-related health information, risk modification suggestions, and motivational materials via either an oral session or a booklet. The two control groups were provided general health information via either an oral session or a booklet. At 12 months follow-up, the treatment groups showed a significant reduction in the frequency of engaging in deep inhalation, breathholding while smoking cannabis, and in driving after cannabis use, compared to their respective control groups. While these risk factors were reduced, no changes were seen in the overall frequency of cannabis use in any group [68].

Also, an RCT was conducted using a web-based intervention based on principles of CBT and MI to reduce the frequency of cannabis use. The study included 225 treatment-seeking participants who were randomized into a webbased treatment group and a web-based control group that contained information about cannabis [69]. At 6 weeks post-treatment, the intervention group reported fewer days of cannabis use, lower quantity of cannabis consumed, and fewer cannabis use disorder symptoms. However, the between-group difference in quantity of cannabis use was no longer significant at 3 months posttreatment.

A small, uncontrolled pilot study evaluated whether aerobic exercise reduced the quantity of cannabis used among individuals with a cannabis use disorder [70]. The treatment lasted 10 days and demonstrated that, after an observed moderate daily exercise regimen, the daily average self-reported cannabis use decreased from mean $=5.9(\mathrm{sd}=3.1)$ to mean $=2.8(\mathrm{sd}=1.6)$ joints per day. In the immediate post-exercise time, cannabis craving scores were also significantly reduced. However, the amount of cannabis use increased to 4.1 $(\mathrm{sd}=2.5)$ joints per day at 2 weeks post-treatment.

The effectiveness of 12-step programs such as Alcoholics (or Narcotics) Anonymous is a controversial subject, with studies and systematic reviews arguing conclusions ranging from beneficial to detrimental outcomes in substance use [71]. However, data suggests that attendance rates at 12-step programs have a dose-response relationship to abstinence and that those who do attend regularly have a greater likelihood of abstinence [71]. We did not find any 12-step studies specifically addressing marijuana use in schizophrenia. However, studies of dual diagnosis populations have suggested that patients with a psychotic disorder have lower attendance rates at 12-step meetings [72]. Adding specialized groups [73] or supplemental treatment such as 12-step facilitation therapy [74] may maximize the effect in a dual diagnosis population.

In summary, psychosocial treatments demonstrated varying degrees of efficacy at treating CUDs in the general population. These results are similar to studies evaluating the treatment of CUDs among schizophrenia patients and, as such, should be considered for treating cannabis use in this population. In 
particular, using aerobic exercise in patients with psychosis could be advantageous due to the known adverse weight and metabolic effects of antipsychotic medications. One of the challenges, both in the general population and among schizophrenia patients, is the tendency for cannabis users to regress towards baseline patterns of use after treatment ends. Overall, this research suggests that treatment may be efficacious at reducing cannabis use or lessening the effects of withdrawal, but only while actively receiving treatment, as post-treatment cannabis use appears to regress towards pre-treatment levels. Thus, continued treatment may be necessary to sustain reduced levels of cannabis use.

The use of pharmacology to treat CUDs in the general population has received much more attention than using this approach to treat CUDs among schizophrenia patients. However, the efficacy of these treatments has been inconsistent. Most trials suffer from low power due to small sample sizes and attrition. Previous studies on CUD treatment have investigated divalproex sodium, buspirone, nefazodone, bupropion SR, and atomoxetine, but they have not yet demonstrated a significant effect by reducing cannabis use or increasing abstinence [75-78].

Two recent RCTs show promise for pharmacological treatment of CUD. One RCT studied $\mathrm{N}$-acetylcysteine (NAC), which is a prodrug of the natural occur-

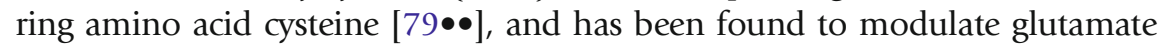
activity in drug-seeking behavior in animals [80]. NAC was recently investigated as a treatment or adjunctive treatment for numerous psychiatric and behavioral conditions including trichotillomania, obsessive compulsive disorder, mood disorders, schizophrenia, and substance use disorders [81]. The efficacy of NAC as a treatment for CUD was evaluated in a double-blinded RCT among individuals with CUDs $(n=116)$ during an 8 -week treatment study. The results indicated the treatment group was twice as likely to produce a negative urine test for cannabinoid compared to the placebo group (OR=2.4, $95 \% \mathrm{CI}=1.1-$ 5.2). At the end of treatment, the NAC group had $40.9 \%$ negative urine cannabinoid tests compared to $27.2 \%$ in the placebo group. At the 4 weeks post-treatment follow-up these rates were 19.0 vs. $10.3 \%$, respectively, but the

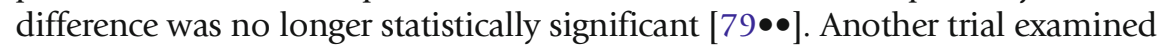
treatment with gabapentin in a phase II double-blinded, placebo-controlled RCT of 50 subjects over 12 weeks and demonstrated that participants receiving active treatment had greater reductions in cannabis use, faster relief of withdrawal symptoms, and improved cognitive function compared to the placebo group [82].

Other recent RCTs testing pharmacologic agents have shown minimal efficacy at treating CUDs compared to placebos [83-85]. A double-blinded RCT evaluated the efficacy of whether the antidepressant venlafaxine extended release (ER)+CBT compared to placebo+CBT reduced cannabis use in addition to depressive symptoms among individuals with co-occurring major depressive disorder and CUD $(n=103)$ [83]. However, the results indicated that the placebo group had improved abstinence over the venlafaxine ER group. A separate RCT investigated the antidepressant fluoxetine in a depressed population and did not find between-group differences in cannabis use compared to 
placebo after 12 weeks of treatment [84]. A pilot RCT evaluated whether CBT in combination with the nicotine patch was efficacious at reducing cannabis use among smokers, but no between-group differences regarding cannabis use were observed after 10 weeks of treatment [85].

Agonist replacement therapy has been effective for treating nicotine dependence (transdermal patch, nicotine replacement gum) [86] and opioid dependence (methadone, buprenorphine) [87, 88]. Hence, a THC agonist or partial agonist could be an effective treatment of cannabis use disorders. For example, dronabinol is an oral formulation of synthetic THC that has been evaluated in two treatment studies. Although both studies did not observe reduced cannabis use, they observed reductions in withdrawal symptoms [89, 90]. Similarly, a recent medication derived from the cannabis sativa plant, nabixmols, contains THC, cannabidiol, and terpenoids. A double-blinded RCT evaluating the efficacy of nabixmols among cannabis dependent inpatients undergoing detoxification found that treatment with the study drug was associated with reduced withdrawal symptoms compared to placebo during a 6-day active treatment phase. However, after 28 days follow-up, changes in cannabis use did not differ between the treatment groups [91].

In summary, there is minimal evidence to support treating CUDs with pharmacotherapy among individuals who are otherwise psychiatrically healthy. Traditional psychotropic medications have not demonstrated a clear effect at reducing cannabis use. The current research on agonist replacement for cannabis has shown efficacy towards withdrawal symptoms, but not towards reducing cannabis use. The effects of NAC and gabapentin are promising and may be beneficial for individuals with mental illnesses such as schizophrenia. However, as with any treatment showing initial potential in the more general CUD population, more research is required to confirm the preliminary results, to optimize protocols, and then to apply and confirm benefits for schizophrenia patients with CUD. Similar to psychosocial interventions, pharmacological treatment may require administration over an extended timeframe in order to achieve a long-term decrease in cannabis use.

\section{Conclusions}

Although a significant percentage of schizophrenia patients have a CUD, the vast majority of available treatments do not target reducing the use of this substance despite its association with poorer clinical outcomes. To date, the most promising pharmacological outcomes were observed in secondgeneration antipsychotic agents, which are currently the first line of care for schizophrenia. A review of the literature suggests that behavioral and pharmacologic treatment studies of CUD among schizophrenia patients and individuals who are otherwise psychiatrically healthy are beginning to emerge. Some pharmacological compounds may be promising, but additional research is needed to evaluate their efficacy in larger trials. Psychosocial interventions including motivational enhancement therapy, cognitive behavioral therapy, and contingency management are the most well-studied treatments and have demonstrated some efficacy in non-psychotic individuals but require persistent treatment to sustain reduced cannabis use and prolong abstinence. 


\section{Acknowledgments}

The Department of Psychiatry and Behavioral Sciences at Northwestern University Feinberg School of Medicine and the Warren Wright Adolescent Center at Northwestern Medicine's Stone Institute of Psychiatry supported the development of this manuscript.

\section{Compliance with Ethics Guidelines}

\section{Conflict of Interest}

Brian R. Schultz declares that he has no conflict of interest.

Lisette Rodriguez-Cabezas declares that she has no conflict of interest.

Dan Angres declares that he has no conflict of interest.

Matthew J. Smith declares that he has no conflict of interest.

\section{Human and Animal Rights and Informed Consent}

All procedures performed in studies involving human participants were in accordance with the ethical standards of the institutional and/or national research committee and with the 1964 Helsinki declaration and its later amendments or comparable ethical standards.

\section{References}

Papers of particular interest, published recently, have been highlighted as:

- Of importance

-• Of major importance

1. Regier DA, Farmer ME, Rae DS, Locke BZ, Keith SJ, Judd LL, et al. Comorbidity of mental disorders with alcohol and other drug abuse. Results from the epidemiologic catchment area (ECA) study. JAMA: J Am Med Assoc. 1990;264(19):2511-8.

2. Talamo A, Centorrino F, Tondo L, Dimitri A, Hennen J, Baldessarini RJ. Comorbid substance-use in schizophrenia: relation to positive and negative symptoms. Schizophr Res. 2006;86(1-3):251-5. doi:10.1016/j. schres.2006.04.004.

3. Barnes TR, Mutsatsa SH, Hutton SB, Watt HC, Joyce EM. Comorbid substance use and age at onset of schizophrenia. Br J Psychiatry J Ment Sci. 2006;188:237-42. doi:10.1192/bjp.bp.104.007237.

4. Potvin S, Pampoulova T, Mancini-Marie A, Lipp O, Bouchard RH, Stip E. Increased extrapyramidal symptoms in patients with schizophrenia and a comorbid substance use disorder. J Neurol Neurosurg Psychiatry. 2006;77(6):796-8. doi:10. 1136/jnnp.2005.079228.

5. Mueser KT, Drake RE, Wallach MA. Dual diagnosis: a review of etiological theories. Addict Behav. 1998;23(6):717-34.

6. Potvin S, Sepehry AA, Stip E. A meta-analysis of negative symptoms in dual diagnosis schizophrenia.
Psychol Med. 2006;36(4):431-40. doi:10.1017/ S003329170500574X.

7. Janssen B, Gaebel W, Haerter M, Komaharadi F, Lindel B, Weinmann S. Evaluation of factors influencing medication compliance in inpatient treatment of psychotic disorders. Psychopharmacology. 2006;187(2):229-36. doi:10.1007/s00213-006-04134.

8. Addington J, Addington D. Substance abuse and cognitive functioning in schizophrenia. J Psychiatry Neurosci: JPN. 1997;22(2):99-104.

9. Swanson JW, Swartz MS, Van Dorn RA, Elbogen EB, Wagner HR, Rosenheck RA, et al. A national study of violent behavior in persons with schizophrenia. Arch Gen Psychiatry. 2006;63(5):490-9. doi:10.1001/ archpsyc.63.5.490.

10. Swartz MS, Wagner HR, Swanson JW, Stroup TS, McEvoy JP, Canive JM, et al. Substance use in persons with schizophrenia: baseline prevalence and correlates from the NIMH CATIE study. J Nerv Ment Dis. 2006;194(3):164-72. doi:10.1097/01.nmd. 0000202575.79453.6e.

11. Koola MM, Gorelick DA, McMahon RP, Liu F, Huestis MA, Linthicum J, et al. Psychiatric symptom differences in people with schizophrenia associated with 
substantial lifetime substance use but no current substance use disorder. Schizophr Res. 2014;152(1):3156. doi:10.1016/j.schres.2013.11.035.

12. Green B, Young R, Kavanagh D. Cannabis use and misuse prevalence among people with psychosis. $\mathrm{Br} \mathrm{J}$ Psychiatry J Ment Sci. 2005;187:306-13. doi:10.1192/ bjp.187.4.306.

13. Ringen PA, Lagerberg TV, Birkenaes AB, Engn J, Faerden A, Jonsdottir $\mathrm{H}$, et al. Differences in prevalence and patterns of substance use in schizophrenia and bipolar disorder. Psychol Med. 2008;38(9):1241-9. doi:10. 1017/S003329170700236X.

14. Coldham EL, Addington J, Addington D. Medication adherence of individuals with a first episode of psychosis. Acta Psychiatr Scand. 2002;106(4):286-90.

15. Fenton WS, Blyler CR, Heinssen RK. Determinants of medication compliance in schizophrenia: empirical and clinical findings. Schizophr Bull. 1997;23(4):63751.

16. Kamali M, Kelly BD, Clarke M, Browne S, Gervin M, Kinsella A, et al. A prospective evaluation of adherence to medication in first episode schizophrenia. Eur Psychiatr: J Assoc Eur Psychiatr. 2006;21(1):29-33. doi:10.1016/j.eurpsy.2005.05.015.

17. Olfson M, Mechanic D, Hansell S, Boyer CA, Walkup J, Weiden PJ. Predicting medication noncompliance after hospital discharge among patients with schizophrenia. Psychiatr Serv. 2000;51(2):216-22.

18. Wilk J, Marcus SC, West J, Countis L, Hall R, Regier DA, et al. Substance abuse and the management of medication nonadherence in schizophrenia. J Nerv Ment Dis. 2006;194(6):454-7. doi:10.1097/01.nmd. 0000221289.54911 .63 .

19. Caspari D. Cannabis and schizophrenia: results of a follow-up study. Eur Arch Psychiatry Clin Neurosci. 1999;249(1):45-9.

20. Hides L, Dawe S, Kavanagh DJ, Young RM. Psychotic symptom and cannabis relapse in recent-onset psychosis. Prospective study. Br J Psychiatry J Ment Sci. 2006;189:137-43. doi:10.1192/bjp.bp.105.014308.

21. Linszen DH, Dingemans PM, Lenior ME. Cannabis abuse and the course of recent-onset schizophrenic disorders. Arch Gen Psychiatry. 1994;51(4):273-9.

22. van Os J, Bak M, Hanssen M, Bijl RV, de Graaf R, Verdoux H. Cannabis use and psychosis: a longitudinal population-based study. Am J Epidemiol.

2002;156(4):319-27.

23. Rais M, van Haren NE, Cahn W, Schnack HG, Lepage C, Collins L, et al. Cannabis use and progressive cortical thickness loss in areas rich in CB1 receptors during the first 5 years of schizophrenia. Eur Neuropsychopharmacol: J Eur Coll

Neuropsychopharmacol. 2010;20(12):855-65. doi:10.1016/j.euroneuro.2010.08.008.

24. Smith MJ, Cobia DJ, Wang L, Alpert KI, Cronenwett WJ, Goldman MB, et al. Cannabis-related working memory deficits and associated subcortical morphological differences in healthy individuals and schizophrenia subjects. Schizophr Bull. 2014;40(2):287-99. doi:10. 1093/schbul/sbt176.

25. Thoma P, Daum I. Comorbid substance use disorder in schizophrenia: a selective overview of neurobiological and cognitive underpinnings. Psychiatry Clin Neurosci. 2013;67(6):367-83. doi:10.1111/pcn.12072.

26. American Psychiatric Association. Diagnostic and statistical manual of mental disorders, Text revision. 4th ed. Washington, DC: American Psychiatric Association; 2000.

27. American Psychiatric Association. Diagnostic and statistical manual of mental disorders. 5th ed. Arlington, VA: American Psychiatric Association; 2013.

28. Hjorthoj C, Fohlmann A, Nordentoft M. Reprint of "Treatment of cannabis use disorders in people with schizophrenia spectrum disorders-a systematic review". Addict Behav. 2009;34(10):846-51. doi:10. 1016/j.addbeh.2009.02.003.

29. Center for Substance Abuse Treatment. Chapter 3-Motivational Interviewing as a Counseling Style. In: Enhancing Motivation for Change in Substance Abuse Treatment. Substance Abuse and Mental Health Services Administration (US), Rockville (MD). 1999. http://www.ncbi.nlm.nih.gov/books/ NBK64964/. Accessed 15 Nov 2014.

30. Beck AT. Thinking and depression. II. Theory and therapy. Arch Gen Psychiatry. 1964;10:561-71.

31. Beck JS, Beck JS. Cognitive behavior therapy: basics and beyond. 2nd ed. New York: Guilford Press; 2011.

32. National Institute on Drug Abuse. Principles of drug addiction treatment: a research-based guide. 3rd ed. Rockville, MD: National Institute on Drug Abuse, National Institutes of Health, U.S. Department of Health and Human Services; 2012. p. vi, 76 pages.

33. Hunt GE, Siegfried N, Morley K, Sitharthan T, Cleary M. Psychosocial interventions for people with both severe mental illness and substance misuse. Cochrane Database Syst Rev. 2013;10, CD001088. doi:10.1002/ 14651858.CD001088.pub3.

This is a Cochrane Database systematic review of evidence for psychosocial interventions in the dual diagnosis population, suggesting a lack of evidence thus far of substance use in people with serious mental illness.

34. Bonsack C, Gibellini Manetti S, Favrod J, Montagrin Y, Besson J, Bovet $\mathrm{P}$, et al. Motivational intervention to reduce cannabis use in young people with psychosis: a randomized controlled trial. Psychother Psychosom. 2011;80(5):287-97. doi:10.1159/000323466.

35. Madigan K, Brennan D, Lawlor E, Turner N, Kinsella A, $\mathrm{O}^{\prime}$ Connor JJ, et al. A multi-center, randomized controlled trial of a group psychological intervention for psychosis with comorbid cannabis dependence over the early course of illness. Schizophr Res.

2013;143(1):138-42. doi:10.1016/j.schres.2012.10. 018.

36. Hjorthoj CR, Fohlmann A, Larsen AM, Gluud C, Arendt M, Nordentoft M. Specialized psychosocial treatment plus treatment as usual (TAU) versus TAU for patients 
with cannabis use disorder and psychosis: the CapOpus randomized trial. Psychol Med. 2013;43(7):1499-510. doi:10.1017/ S0033291712002255.

37. Hjorthoj CR, Orlovska S, Fohlmann A, Nordentoft M. Psychiatric treatment following participation in the CapOpus randomized trial for patients with comorbid cannabis use disorder and psychosis. Schizophr Res. 2013;151(1-3):191-6. doi:10.1016/ j.schres.2013.10.014.

38. Smeerdijk M, Keet R, Dekker N, van Raaij B, Krikke M, Koeter $\mathrm{M}$, et al. Motivational interviewing and interaction skills training for parents to change cannabis use in young adults with recent-onset schizophrenia: a randomized controlled trial. Psychol Med.

2012;42(8):1627-36. doi:10.1017/

S0033291711002832.

39. Koola MM, Boggs DL, Kelly DL, Liu F, Linthicum JA, Turner HE, et al. Relief of cannabis withdrawal symptoms and cannabis quitting strategies in people with schizophrenia. Psychiatry Res. 2013;209(3):273-8. doi:10.1016/j.psychres.2013.07.044.

40. Lehman AF, Lieberman JA, Dixon LB, McGlashan TH, Miller AL, Perkins DO, et al. Practice guideline for the treatment of patients with schizophrenia, second edition. Am J Psychiatr. 2004;161(2 Suppl):1-56.

41. Working Group of the Clinical Practice Guideline for Schizophrenia and Incipient Psychotic Disorder, Mental Health Forum, coordination. Clinical Practice Guideline for Schizophrenia and Incipient Psychotic Disorder. Quality Plan for the National Health System of the Ministry of Health and Consumer Affairs. Madrid: Agency for Health Technology Assessment and Research; 2009.

42. Potvin S, Stip E, Lipp O, Elie R, Mancini-Marie A, Demers $\mathrm{MF}$, et al. Quetiapine in patients with comorbid schizophrenia-spectrum and substance use disorders: an open-label trial. Curr Med Res Opin. 2006;22(7):1277-85. doi:10.1185/ 030079906X112561.

43. Zimmet SV, Strous RD, Burgess ES, Kohnstamm S, Green AI. Effects of clozapine on substance use in patients with schizophrenia and schizoaffective disorder: a retrospective survey. J Clin Psychopharmacol. 2000;20(1):94-8.

44. van Nimwegen LJ, de Haan L, van Beveren NJ, van der Helm M, van den Brink W, Linszen D. Effect of olanzapine and risperidone on subjective wellbeing and craving for cannabis in patients with schizophrenia or related disorders: a double-blind randomized controlled trial. Can J Psychiatry. 2008;53(6):400-5.

45. Sevy S, Robinson DG, Sunday S, Napolitano B, Miller $\mathrm{R}, \mathrm{McCormack} \mathrm{J}$, et al. Olanzapine vs. risperidone in patients with first-episode schizophrenia and a lifetime history of cannabis use disorders: 16 weeks clinical and substance use outcomes. Psychiatry Res.

2011;188(3):310-4. doi:10.1016/j.psychres.2011.05. 001.
46. Machielsen MW, Veltman DJ, van den Brink W, de Haan L. The effect of clozapine and risperidone on attentional bias in patients with schizophrenia and a cannabis use disorder: an fMRI study. J Psychopharmacol. 2014;28(7):633-42. doi:10.1177/ 0269881114527357.

This is a randomized trial in a dual diagnosis population showing decreased cannabis craving when treated with clozapine vs. risperidone, and showing corresponding changes in insula activation.

47. Machielsen M, Beduin AS, Dekker N, Genetic R, Outcome of Psychosis I, Kahn RS, et al. Differences in craving for cannabis between schizophrenia patients using risperidone, olanzapine or clozapine. J Psychopharmacol. 2012;26(1):189-95. doi:10.1177/ 0269881111408957.

48. Komossa K, Rummel-Kluge C, Hunger H, Schmid F, Schwarz S, Duggan L, et al. Olanzapine versus other atypical antipsychotics for schizophrenia. Cochrane Database Syst Rev. 2010;3, CD006654. doi:10.1002/ 14651858.CD006654.pub2.

49. Leucht S, Cipriani A, Spineli L, Mavridis D, Orey D, Richter F, et al. Comparative efficacy and tolerability of 15 antipsychotic drugs in schizophrenia: a multipletreatments meta-analysis. Lancet.

2013;382(9896):951-62. doi:10.1016/S01406736(13)60733-3.

50. Sinha R, Easton C, Renee-Aubin L, Carroll KM. Engaging young probation-referred marijuana-abusing individuals in treatment: a pilot trial. Am J Addict Am Acad Psychiatr Alcohol Addict. 2003;12(4):314-23.

51. Carroll KM, Easton CJ, Nich C, Hunkele KA, Neavins $\mathrm{TM}$, Sinha R, et al. The use of contingency management and motivational/skills-building therapy to treat young adults with marijuana dependence. J Consult Clin Psychol. 2006;74(5):955-66. doi:10.1037/0022006X.74.5.955.

52. Kadden RM, Litt MD, Kabela-Cormier E, Petry NM. Abstinence rates following behavioral treatments for marijuana dependence. Addict Behav. 2007;32(6):1220-36. doi:10.1016/j.addbeh.2006.08. 009.

53. Marijuana Treatment Project Research G. Brief treatments for cannabis dependence: findings from a randomized multisite trial. J Consult Clin Psychol. 2004;72(3):455-66. doi:10.1037/0022-006X.72.3. 455.

54. Budney AJ, Higgins ST, Radonovich KJ, Novy PL. Adding voucher-based incentives to coping skills and motivational enhancement improves outcomes during treatment for marijuana dependence. J Consult Clin Psychol. 2000;68(6):1051-61.

55. Budney AJ, Moore BA, Rocha HL, Higgins ST. Clinical trial of abstinence-based vouchers and cognitivebehavioral therapy for cannabis dependence. J Consult Clin Psychol. 2006;74(2):307-16. doi:10.1037/0022006X.4.2.307.

56. Copeland J, Swift W, Roffman R, Stephens R. A randomized controlled trial of brief cognitive-behavioral 
interventions for cannabis use disorder. J Subst Abus Treat. 2001;21(2):55-64.

discussion 5-6.

57. Stephens RS, Roffman RA, Simpson EE. Treating adult marijuana dependence: a test of the relapse prevention model. J Consult Clin Psychol. 1994;62(1):92-9.

58. Stephens RS, Roffman RA, Curtin L. Comparison of extended versus brief treatments for marijuana use. J Consult Clin Psychol. 2000;68(5):898-908.

59. Litt MD, Kadden RM, Petry NM. Behavioral treatment for marijuana dependence: randomized trial of contingency management and self-efficacy enhancement. Addict Behav. 2013;38(3):1764-75. doi:10.1016/j. addbeh.2012.08.011.

60. Carroll KM, Nich C, Lapaglia DM, Peters EN, Easton CJ, Petry NM. Combining cognitive behavioral therapy and contingency management to enhance their effects in treating cannabis dependence: less can be more, more or less. Addiction. 2012;107(9):1650-9. doi:10. 1111/j.1360-0443.2012.03877.x.

61. Hoch E, Noack R, Henker J, Pixa A, Hofler M, Behrendt $S$, et al. Efficacy of a targeted cognitive-behavioral treatment program for cannabis use disorders (CANDIS). Eur Neuropsychopharmacol: J Eur Coll Neuropsychopharmacol. 2012;22(4):267-80. doi:10. 1016/j.euroneuro.2011.07.014.

62.• Hoch E, Buhringer G, Pixa A, Dittmer K, Henker J, Seifert A, et al. CANDIS treatment program for cannabis use disorders: findings from a randomized multisite translational trial. Drug Alcohol Depend. 2014;134:185-93. doi:10.1016/j.drugalcdep.2013.09. 028.

This is an RCT combining motivational enhancement therapy, cognitive behavioral therapy, and problem-solving training showing significant decrease in cannabis use during active treatment.

63. Walker DD, Stephens R, Roffman R, Demarce J, Lozano $\mathrm{B}$, Towe S, et al. Randomized controlled trial of motivational enhancement therapy with nontreatmentseeking adolescent cannabis users: a further test of the teen marijuana check-up. Psychol Addict Behav: J Soc Psychol Addict Behav. 2011;25(3):474-84. doi:10. 1037/a0024076.

64. Liddle HA. Treating adolescent substance abuse using multidimensional family therapy. In: Weisz J, Kazdin A, editors. Evidence-based psychotherapies for children and adolescents. 2nd ed. New York: Guilford Press; 2010. p. 416-32.

65. Hendriks V, van der Schee E, Blanken P. Treatment of adolescents with a cannabis use disorder: main findings of a randomized controlled trial comparing multidimensional family therapy and cognitive behavioral therapy in the Netherlands. Drug Alcohol Depend. 2011;119(1-2):64-71. doi:10.1016/j.drugalcdep. 2011.05.021.

66. Hendriks V, van der Schee E, Blanken P. Matching adolescents with a cannabis use disorder to multidimensional family therapy or cognitive behavioral therapy: treatment effect moderators in a randomized controlled trial. Drug Alcohol Depend. 2012;125(12):119-26. doi:10.1016/j.drugalcdep.2012.03.023.

67. Rigter H, Henderson CE, Pelc I, Tossmann P, Phan O, Hendriks $\mathrm{V}$, et al. Multidimensional family therapy lowers the rate of cannabis dependence in adolescents: a randomised controlled trial in Western European outpatient settings. Drug Alcohol Depend.

2013;130(1-3):85-93. doi:10.1016/j.drugalcdep.2012. 10.013 .

This is an intent-to-treat randomized study in adolescents comparing MDFT to individual psychotherapy, with a greater decline in rates of cannabis dependence in the MDFT group.

68. Fischer B, Jones W, Shuper P, Rehm J. 12-month follow-up of an exploratory 'brief intervention' for highfrequency cannabis users among Canadian university students. Subst Abus Treat Prev Policy. 2012;7:15. doi:10.1186/1747-597X-7-15.

69. Rooke S, Copeland J, Norberg M, Hine D, McCambridge J. Effectiveness of a self-guided webbased cannabis treatment program: randomized controlled trial. J Med Internet Res. 2013;15(2):e26. doi:10.2196/jmir.2256.

70. Buchowski MS, Meade NN, Charboneau E, Park S, Dietrich MS, Cowan RL, et al. Aerobic exercise training reduces cannabis craving and use in non-treatment seeking cannabis-dependent adults. PLoS One. 2011;6(3):e17465. doi:10.1371/journal.pone. 0017465.

71. Kaskutas LA. Alcoholics anonymous effectiveness: faith meets science. J Addict Dis. 2009;28(2):145-57. doi:10.1080/10550880902772464.

72. Bogenschutz MP, Geppert CM, George J. The role of twelve-step approaches in dual diagnosis treatment and recovery. Am J Addict Am Acad Psychiatr Alcohol Addict. 2006;15(1):50-60. doi:10.1080/ 10550490500419060.

73. Laudet AB, Magura S, Cleland CM, Vogel HS, Knight EL, Rosenblum A. The effect of 12-step based fellowship participation on abstinence among dually diagnosed persons: a 2 years longitudinal study. J Psychoactive Drugs. 2004;36(2):207-16. doi:10.1080/ 02791072.2004 .10399731$.

74. Bogenschutz MP, Rice SL, Tonigan JS, Vogel HS, Nowinski J, Hume D, et al. 12-Step facilitation for the dually diagnosed: a randomized clinical trial. J Subst Abus Treat. 2014;46(4):403-11. doi:10.1016/j.jsat. 2013.12.009.

75. Levin FR, McDowell D, Evans SM, Nunes E, Akerele E, Donovan S, et al. Pharmacotherapy for marijuana dependence: a double-blind, placebo-controlled pilot study of divalproex sodium. Am J Addict Am Acad Psychiatr Alcohol Addict. 2004;13(1):21-32.

76. Carpenter KM, McDowell D, Brooks DJ, Cheng WY, Levin FR. A preliminary trial: double-blind comparison of nefazodone, bupropion-SR, and placebo in the treatment of cannabis dependence. Am J Addict Am Acad Psychiatr Alcohol Addict. 2009;18(1):53-64. doi:10.1080/10550490802408936. 
77. McRae-Clark AL, Carter RE, Killeen TK, Carpenter MJ, Wahlquist AE, Simpson SA, et al. A placebocontrolled trial of buspirone for the treatment of marijuana dependence. Drug Alcohol Depend. 2009;105(1-2):132-8. doi:10.1016/j.drugalcdep. 2009.06.022.

78. McRae-Clark AL, Carter RE, Killeen TK, Carpenter MJ, White KG, Brady KT. A placebo-controlled trial of atomoxetine in marijuana-dependent individuals with attention deficit hyperactivity disorder. Am J Addict Am Acad Psychiatr Alcohol Addict. 2010;19(6):481-9. doi:10.1111/j.1521-0391.2010.00076.x.

79.• Gray KM, Carpenter MJ, Baker NL, DeSantis SM, Kryway E, Hartwell KJ, et al. A double-blind randomized controlled trial of $\mathrm{N}$-acetylcysteine in cannabisdependent adolescents. Am J Psychiatr. 2012;169(8):805-12. doi:10.1176/appi.ajp.2012. 12010055 .

This RCT is an initial study using NAC for treating cannabis use, showing an odds ratio $=2.4$ of negative urine cannabinoid test in the active treatment group compared to placebo.

80. Kalivas PW, Volkow ND. New medications for drug addiction hiding in glutamatergic neuroplasticity. Mol Psychiatry. 2011;16(10):974-86. doi:10.1038/mp. 2011.46.

81. Dean O, Giorlando F, Berk M. N-acetylcysteine in psychiatry: current therapeutic evidence and potential mechanisms of action. J Psychiatry Neurosci: JPN. 2011;36(2):78-86. doi:10.1503/jpn.100057.

82. Mason BJ, Crean R, Goodell V, Light JM, Quello S, Shadan F, et al. A proof-of-concept randomized controlled study of gabapentin: effects on cannabis use, withdrawal and executive function deficits in cannabisdependent adults. Neuropsychopharmacol: Off Pub Am Coll Neuropsychopharmacol. 2012;37(7):168998. doi:10.1038/npp.2012.14.

83. Levin FR, Mariani J, Brooks DJ, Pavlicova M, Nunes EV, Agosti V, et al. A randomized double-blind, placebocontrolled trial of venlafaxine-extended release for $\mathrm{CO}-$ occurring cannabis dependence and depressive disorders. Addiction. 2013;108(6):1084-94. doi:10.1111/ add.12108.
84. Cornelius JR, Bukstein OG, Douaihy AB, Clark DB, Chung TA, Daley DC, et al. Double-blind fluoxetine trial in comorbid MDD-CUD youth and young adults. Drug Alcohol Depend. 2010;112(1-2):39-45. doi:10. 1016/j.drugalcdep.2010.05.010.

85. Hill KP, Toto LH, Lukas SE, Weiss RD, Trksak GH, Rodolico JM, et al. Cognitive behavioral therapy and the nicotine transdermal patch for dual nicotine and cannabis dependence: a pilot study. Am J Addict Am Acad Psychiatr Alcohol Addict. 2013;22(3):233-8. doi:10.1111/j.1521-0391.2012.12007.x.

86. Stead LF, Perera R, Bullen C, Mant D, Hartmann-Boyce J, Cahill K, et al. Nicotine replacement therapy for smoking cessation. Cochrane Database Syst Rev. 2012;11, CD000146. doi:10.1002/14651858. CD000146.pub4.

87. Mattick RP, Breen C, Kimber J, Davoli M. Methadone maintenance therapy versus no opioid replacement therapy for opioid dependence. Cochrane Database Syst Rev. 2009;3, CD002209. doi:10.1002/14651858. CD002209.pub2.

88. Mattick RP, Breen C, Kimber J, Davoli M. Buprenorphine maintenance versus placebo or methadone maintenance for opioid dependence. Cochrane Database Syst Rev. 2014;2, CD002207. doi:10.1002/ 14651858.CD002207.pub4.

89. Levin FR, Mariani JJ, Brooks DJ, Pavlicova M, Cheng W, Nunes EV. Dronabinol for the treatment of cannabis dependence: a randomized, double-blind, placebocontrolled trial. Drug Alcohol Depend. 2011;116(13):142-50. doi:10.1016/j.drugalcdep.2010.12.010.

90. Vandrey R, Stitzer ML, Mintzer MZ, Huestis MA, Murray JA, Lee $D$. The dose effects of short-term dronabinol (oral THC) maintenance in daily cannabis users. Drug Alcohol Depend. 2013;128(1-2):64-70. doi:10.1016/ j.drugalcdep.2012.08.001.

91. Allsop DJ, Copeland J, Lintzeris N, Dunlop AJ, Montebello M, Sadler C, et al. Nabiximols as an agonist replacement therapy during cannabis withdrawal: a randomized clinical trial. JAMA Psychiatr. 2014;71(3):281-91. doi:10.1001/jamapsychiatry. 2013.3947. 